Topological Methods in Nonlinear Analysis

Volume 46, No. 2, 2015, 1013-1028

DOI: 10.12775 TMNA 2015.075

(C) 2015 Juliusz Schauder Centre for Nonlinear Studies

Nicolaus Copernicus University

\title{
TOPOLOGICAL AND MEASURE PROPERTIES OF SOME SELF-SIMILAR SETS
}

\author{
Taras BanakH - Artur Bartoszewicz \\ MaŁgorzata FilipczaK — EMilia SZyMONIK
}

\begin{abstract}
Given a finite subset $\Sigma \subset \mathbb{R}$ and a positive real number $q<1$ we study topological and measure-theoretic properties of the self-similar set $K(\Sigma ; q)=\left\{\sum_{n=0}^{\infty} a_{n} q^{n}:\left(a_{n}\right)_{n \in \omega} \in \Sigma^{\omega}\right\}$, which is the unique compact solution of the equation $K=\Sigma+q K$. The obtained results are applied to studying partial sumsets $E(x)=\left\{\sum_{n=0}^{\infty} x_{n} \varepsilon_{n}:\left(\varepsilon_{n}\right)_{n \in \omega} \in\{0,1\}^{\omega}\right\}$ of multigeometric sequences $x=\left(x_{n}\right)_{n \in \omega}$. Such sets were investigated by Ferens, Morán, Jones and others. The aim of the paper is to unify and deepen existing piecemeal results.
\end{abstract}

\section{Introduction}

Suppose that $x=\left(x_{n}\right)_{n=1}^{\infty}$ belongs to $l_{1} \backslash c_{00}$ which means that $x$ is an absolutely summable sequence with infinitely many nonzero terms. Let

$$
E(x)=\left\{\sum_{n=1}^{\infty} \varepsilon_{n} x_{n}:\left(\varepsilon_{n}\right)_{n=1}^{\infty} \in\{0,1\}^{\mathbb{N}}\right\}
$$

denotes the set of all subsums of the series $\sum_{n=1}^{\infty} x_{n}$, called the achievement set (or a partial sumset) of $x$. The investigation of topological properties of achievement

2010 Mathematics Subject Classification. 40A05, 28A80, 11K31.

Key words and phrases. Self-similar set, multigeometric sequence, Cantorval.

The first author has been partially financed by NCN grant DEC-2012/07/D/ST1/02087. 
sets was initiated almost one hundred years ago. In 1914 Soichi Kakeya [8] presented the following result:

Theorem 1.1 (Kakeya). For any sequence $x \in l_{1} \backslash c_{00}$

(a) $E(x)$ is a perfect compact set.

(b) If $\left|x_{n}\right|>\sum_{i>n}\left|x_{i}\right|$ for almost all $n$, then $E(x)$ is homeomorphic to the ternary Cantor set.

(c) If $\left|x_{n}\right| \leq \sum_{i>n}\left|x_{i}\right|$ for almost all $n$, then $E(x)$ is a finite union of closed intervals. In the case of non-increasing sequence $x$, the last inequality is also necessary for $E(x)$ to be a finite union of intervals.

Moreover, Kakeya conjectured that $E(x)$ is either nowhere dense or a finite union of intervals. Probably, the first counterexample to this conjecture was given by Weinstein and Shapiro ([16]) and, independently, by Ferens ([5]). The simplest example was presented by Guthrie and Nymann [6]: for the sequence $c=\left(\left(5+(-1)^{n}\right) / 4^{n}\right)_{n=1}^{\infty}$, the set $T=E(c)$ contains an interval but is not a finite union of intervals. In the same paper they formulated the following theorem, finally proved in [12]:

Theorem 1.2. For any sequence $x \in l_{1} \backslash c_{00}, E(x)$ is one of the following sets:

(a) a finite union of closed intervals;

(b) homeomorphic to the Cantor set;

(c) homeomorphic to the set $T$.

Note that the set $T=E(c)$ is homeomorphic to $C \cup \bigcup_{n=1}^{\infty} S_{2 n-1}$, where $S_{n}$ denotes the union of the $2^{n-1}$ open middle thirds which are removed from $[0,1]$ at the $n$-th step in the construction of the Cantor ternary set $C$. Such sets are called Cantorvals (to emphasize their similarity to unions of intervals and to the Cantor set simultaneously). Formally, a Cantorval (more precisely, an $\mathcal{M}$ Cantorval, see [9]) is a non-empty compact subset $S$ of the real line such that $S$ is the closure of its interior, and both endpoints of any component with non-empty interior are accumulation points of one-point components of $S$. A non-empty subset $C$ of the real line $\mathbb{R}$ will be called a Cantor set if it is compact, zerodimensional, and has no isolated points.

Let us observe that Theorem 1.2 says that $l_{1}$ can be divided into 4 sets: $c_{00}$ and the sets connected with cases (a), (b) and (c). Some algebraic and topological properties of these sets have been recently considered in [1].

We will describe sequences constructed by Weinstein and Shapiro, Ferens and Guthrie and Nymann using the notion of multigeometric sequence. We call 